\title{
Visualization and Classification of Deeply Seated Collateral Networks in Moyamoya Angiopathy with 7T MRI
}

\author{
(D) T. Matsushige, (D) M. Kraemer, (D). Sato, (DP. Berlit, (D) M. Forsting, (D) M.E. Ladd, (D) R. Jabbarli, (D). Sure, (D) N. Khan,
}

(D) M. Schlamann, and (D).H. Wrede

\begin{abstract}
BACKGROUND AND PURPOSE: Collateral networks in Moyamoya angiopathy have a complex angioarchitecture difficult to comprehend on conventional examinations. This study aimed to evaluate morphologic patterns and the delineation of deeply seated collateral networks using ultra-high-field MRA in comparison with conventional DSA.
\end{abstract}

MATERIALS AND METHODS: Fifteen white patients with Moyamoya angiopathy were investigated in this prospective trial. Sequences acquired at 7T were TOF-MRA with $0.22 \times 0.22 \times 0.41 \mathrm{~mm}^{3}$ resolution and MPRAGE with $0.7 \times 0.7 \times 0.7 \mathrm{~mm}^{3}$ resolution. Four raters evaluated the presence of deeply seated collateral networks and image quality in a consensus reading of DSA, TOF-MRA, and MPRAGE using a 5-point scale in axial source images and maximum intensity projections. Delineation of deeply seated collateral networks by different imaging modalities was compared by means of the McNemar test, whereas image quality was compared using the Wilcoxon signed-rank test.

RESULTS: The relevant deeply seated collateral networks were classified into 2 categories and 6 pathways. A total of 100 collateral networks were detected on DSA; 106, on TOF-MRA; and 73, on MPRAGE. Delineation of deeply seated collateral networks was comparable between TOF-MRA and DSA $(P=.25)$; however, both were better than MPRAGE $(P<.001)$.

CONCLUSIONS: This study demonstrates excellent delineation of 6 distinct deeply seated collateral network pathways in Moyamoya angiopathy in white adults using 7T TOF-MRA, comparable to DSA.

ABBREVIATIONS: DSCN = deeply seated collateral network; $M M A=$ Moyamoya angiopathy; $\mathrm{SEM}=$ standard error of the mean

M oyamoya angiopathy (MMA) is a rare cerebral vasculopathy with progressive steno-occlusion of the intracranial portion of the internal carotid arteries. ${ }^{1,2}$ A secondary crucial pathophysiology is characterized by progressive formation of col-

Received July 5, 2016; accepted after revision March 7, 2018.

From the Department of Neurosurgery (T.M., T.S., R.J., U.S., K.H.W.), University Hospital Essen, University Duisburg-Essen, Essen, Germany; Department of Neurosurgery (T.M.), Graduate School of Biomedical and Health Sciences, Hiroshima University, Hiroshima, Japan; Erwin L. Hahn Institute for Magnetic Resonance Imaging (T.M., T.S., M.E.L., K.H.W.), University Duisburg-Essen, Essen, Germany; Department of Neurology (M.K., P.B.), Alfried Krupp Hospital, Essen, Germany; Department of Neurology (M.K.), University Hospital Duesseldorf, Duesseldorf, Germany; Department of Neurosurgery (T.S.), Fukushima Medical University, Fukushima, Japan; Department of Diagnostic and Interventional Radiology and Neuroradiology (M.F., M.S.), University Hospital Essen, Essen, Germany; Medical Physics in Radiology (M.E.L.), German Cancer Research Center, Heidelberg, Germany; Faculty of Physics and Astronomy and Faculty of Medicine (M.E.L.), University of Heidelberg, Heidelberg, Germany; Moyamoya Center, Division of Pediatric Neurosurgery (N.K.), Department of Surgery, University Children's Hospital Zurich, Zurich, Switzerland; and Department of Neuroradiology (M.S.), University Hospital Cologne, Cologne, Germany.

Toshinori Matsushige, Markus Kraemer, Marc Schlamann, and Karsten H. Wrede contributed equally to this work.

This work received funding from the University of Duisburg-Essen (Programm zur internen Forschungsförderung Essen [IFORES grant] to K.H.W.). lateral vessels at the base of the brain, so-called "Moyamoya vessels," and mutual anastomoses to compensate hemodynamic insufficiency. ${ }^{1-4}$ Considering these diagnostic criteria, MRA along with flow-void-based T2-weighted imaging, plays a primary role in addition to DSA in the current clinical work-up. ${ }^{2,5}$

Recent reports suggested the importance of collateral networks in MMA for classifying disease severity. ${ }^{6-10}$ Despite these collaterals, MMA manifests with hemodynamic insufficiency with transient ischemic attacks, strokes, and choreatiform movements in early childhood and in young adults. ${ }^{11-13}$ In contrast to the positive compensatory role of collateral networks, clinical presentation with cerebral bleeding has been attributed to the rupture of extensive fragile collateral vessels, and chronic headaches have been attributed to extensive dural anastomoses. ${ }^{14,15}$

Please address correspondence to Karsten $\mathrm{H}$. Wrede, MD, University Hospital Essen, Department of Neurosurgery, Hufelandstr 55, 45147 Essen, Germany; e-mail: karsten.wrede@uk-essen.de

三 Indicates article with supplemental on-line tables.

http://dx.doi.org/10.3174/ajnr.A5700 
While morphologic submillimeter studies of complex and tiny collateral vessels are still ongoing, assessment of these collaterals in most cases remains unfeasible with conventional 3T MR imaging and is challenging with DSA, the current clinical diagnostic standard. Nevertheless, proliferation of these fine anastomoses could be evaluated by MR imaging indirectly as a summation of high signal intensity in the basal ganglia, ${ }^{16,17}$ or, alternatively, by specific collateral pathways running adjacent to the ventricles. ${ }^{9,18}$

Since Cho et $\mathrm{al}^{19}$ reported the application of ultra-high-field 7T MRA for excellent visualization of intracranial perforating arteries, depiction of intracranial submillimeter vessels has further advanced. Several studies have suggested the feasibility and diagnostic accuracy for delineation of submillimeter perforating arteries, microvascular structures, and vessel walls. ${ }^{20-26}$ Therefore, studies on collateral networks in MMA using ultra-high-field 7T MR imaging are warranted. Better visualization of deeply seated collateral networks (DSCNs) in MMA might improve our understanding of this rare and complicated pathophysiology and presumably facilitate treatment decisions. In the present study, morphologic visualization of collateral networks in adult patients with MMA using 7T MRA was evaluated in comparison with DSA. Moreover, this study aimed to delineate different types of collateral networks.

\section{MATERIALS AND METHODS}

The study patients were recruited consecutively at Alfried Krupp Hospital in Essen, Germany. There were no ethnic inclusion or exclusion criteria. All patients with Moyamoya angiopathy in the hospital cohort were of white ethnic background. Participation was voluntary and refusal to participate had no consequence for further treatment. The University of Duisburg-Essen ethics committee authorized the study, and all patients provided written informed consent before examination. The study was conducted according to the principles of the Declaration of Helsinki and was Health Insurance Portability and Accountability Act-compliant.

In addition to a standard clinical work-up with conventional MRA and selective DSA, 15 patients with MMA were prospectively recruited for a 7T TOF-MRA feasibility study commencing in October 2011. ${ }^{27}$ Except in the case of patients with a second postoperative 7T MR imaging, the 7T MR imaging was performed within 1 week of DSA. In the current observational study, delineation of collateral networks has been further evaluated. Inclusion criteria were the following: 1) idiopathic MMA (classic Moyamoya disease and unilateral variants of Moyamoya angiopathy) previously diagnosed via DSA and conventional MR imaging, 2) 18 years of age and older, and 3) the ability to give informed consent. Exclusion criteria were the following: 1) a known concomitant disease (Moyamoya syndrome), ie, cerebral vasculitis, Down syndrome, or von Recklinghausen neurofibromatosis, ${ }^{2} 2$ ) the presence of a cardiac pacemaker or any other electronic implants, 3) pregnancy or breast-feeding, and 4) claustrophobia.

\section{High-Resolution 7T MRA}

All subjects were evaluated using a 7T whole-body MR imaging system (Magnetom 7T; Siemens, Erlangen, Germany) equipped with a 1-channel transmit/32-channel receive head radiofrequency coil (Nova Medical, Wilmington, Massachusetts) begin- ning in October 2011 at the Erwin L. Hahn Institute for Magnetic Resonance Imaging, University of Duisburg-Essen, Essen, Germany. The system has a gradient system providing $40 \mathrm{mT} / \mathrm{m}$ maximum amplitude and a slew rate of $200 \mathrm{mT} / \mathrm{m} / \mathrm{ms}$. High-resolution MRA was acquired with a customized 3D fast low-angle shot TOF pulse sequence ${ }^{27,28}$ (voxel size, $0.22 \times 0.22 \times 0.41 \mathrm{~mm}^{3}$ ) and with a modified MPRAGE sequence ${ }^{29-31}$ (voxel size, $0.7 \times 0.7 \times$ $0.7 \mathrm{~mm}^{3}$ ). Detailed sequence parameters for 7T MR imaging are listed in On-line Table 1.

\section{DSA}

Six-vessel DSA was performed using an Axiom Artis angiography suite (Siemens) in the Department of Neuroradiology of Alfried Krupp Hospital in Essen, Germany. After the standardized introduction, $45^{\circ}$ oblique 2-plane projections of the posterior circulatory system and both internal carotid artery circulation areas were acquired.

\section{Image Analysis}

Image evaluation was performed using a freeware DICOM reader (Horos, Version 2.0.1; http://www.horosproject.org/). DSCNs were classified according to location and connecting vessels, referring to morphologic characteristics described by Baltsavias et $\mathrm{al}^{32-34}$ who described collateral circulation in pediatric Moyamoya disease with superselective angiography. Images were analyzed by 1 experienced vascular neurologist (M.K.), 2 experienced vascular neurosurgeons (T.M. and K.H.W.), and 1 experienced neuroradiologist (M.S.) in a consensus reading. At first, all raters evaluated the images separately, and afterward, a consensus was found by discussion. Reading was performed in 9 sessions with at least a 2-week interval between each session ( 3 sessions for every imaging technique and 5 patients per session).

Overall image quality and image quality for anastomoses to major trunk vessels and collateral pathways to cortical vessels were rated on a 5 -point scale $(5=$ excellent, $4=$ good, $3=$ moderate, 2 = poor, and $1=$ nondiagnostic). Collateral networks were rated by a 3 -stage evaluation process using a different 5 -point scale ( $5=$ excellent, collateral networks obviously detected with clear identification of the vessel course and high vessel-tissue contrast; $4=$ good, collateral networks well-detected with lower vessel-tissue contrast; 3 = moderate, collateral networks partially obscured but still adequate for diagnosis; 2 = poor, collateral connections scarcely demonstrated but sufficient to presume their existence; and $1=$ no collateral networks detected).

At first, each rater individually assessed the DSA images for the presence and image quality of collateral networks. Additionally, the Suzuki stages in DSA were rated by an experienced vascular neurosurgeon as a fifth rater (N.K.). In the second step, the transverse source images and MIPs with slab thicknesses of 10-20 mm obtained with TOF-MRA and MPRAGE were evaluated in the same way. Finally, all 4 raters discussed their individual evaluations to establish a consensus reading. To assess interobserver accordance, an experienced vascular neurosurgeon evaluated the data blinded to the consensus results as a fifth rater (T.S.). For analysis of image quality in collateral networks, subjects without detection on DSA were excluded. 


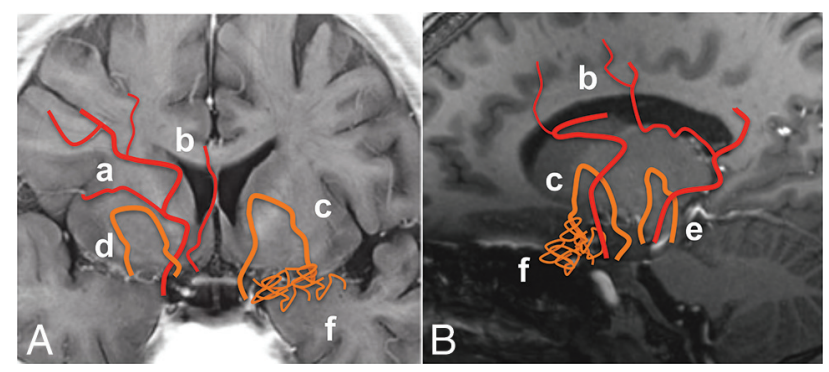

FIG 1. Schematic illustrations of deeply seated collateral networks are shown in coronal $(A)$ and sagittal $(B)$ MR imaging views. Six pathways of collateral vessels according to perfusing territories can be divided into collateral networks to cortical vessels (remote, type a and b) and to major trunk vessels (local, types c-f). Anastomoses between striate arteries or choroidal arteries and cortical arteries directly or via medullary arteries (a); septal transcallosal anastomoses between choroidal arteries with pericallosal arteries (b); anastomoses between choroidal arteries or the posterior communicating artery and thalamostriate arteries (c); intrastriatal anastomosis among striatal arteries (d); intrathalamic anastomosis among thalamic arteries (e); and focal Moyamoya vessels in the basal cistern ( $f$ ).

\section{Statistical Analysis}

Delineation of collateral networks was analyzed by the McNemar test. Image quality was compared using Wilcoxon signed-rank tests. Corrections for multiple comparisons were performed with the Bonferroni method. Interobserver accordance was assessed using the $\kappa$ coefficient. Statistical analyses were performed with the JMP statistical package (Version 10; SAS Institute, Cary, North Carolina) and the STATA software package (Version 14.2; StataCorp, College Station, Texas).

\section{RESULTS}

Patients comprised 4 men and 11 women with an average age of 36 years (range, 19-58 years). Two patients had a unilateral variant of MMA (cases 3 and 4). All were from white family backgrounds. Two patients underwent all examinations both before and up to 6 months after bypass surgery (cases 1 and 5). Clinical characteristics are summarized in On-line Table 2. All examinations were successfully performed without adverse events.

In total, 32 affected hemispheres were evaluated. According to the Suzuki angiographic staging, 7 hemispheres were rated as stage II, 20 were rated as stage III, and 5 were rated as stage IV. Overall image quality was rated excellent for most scans. The mean overall image quality was 4.7 (standard error of the mean $[\mathrm{SEM}]=0.12$; range, $4-5)$ in DSA, $4.9(\mathrm{SEM}=0.07$; range, $4-5)$ in TOF-MRA, and 4.5 (SEM $=0.13$; range, $4-5)$ in MPRAGE.

Interobserver accordance was almost perfect for identification of collateral networks in DSA (0.9001), TOF-MRA (0.9243), and MPRAGE (0.8699). Interobserver accordance for the quality of collateral network delineation was moderate to substantial in DSA (0.4214), TOF-MRA (0.6143), and MPRAGE (0.4825), with only 1-point variability for most differing ratings.

DSCNs were classified into 2 major categories (connection to cortical vessels/major trunk vessels) with a total of 6 pathways (Fig 1, a-f). One hundred collateral networks were detected in DSA; 106, in TOF-MRA; and 73, in MPRAGE. Basic patient characteristics and scoring for DSCNs are summarized in On-line Tables 3 and 4 .

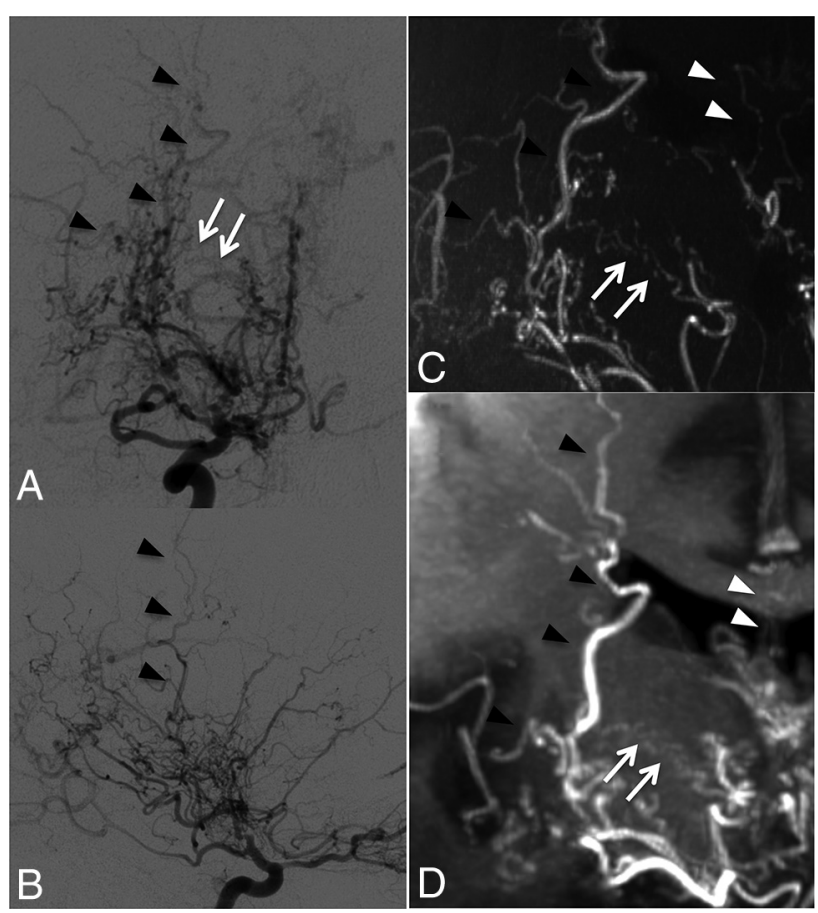

FIG 2. Case 1. Selective right internal carotid angiography (anteroposterior, A; lateral view, B) shows anastomotic connections to cortical arteries from the anterior choroidal artery (black arrowheads, type b) as well as from thalamostriate arteries (white arrows, type a). Collateral networks are excellently visualized in MIP from TOF-MRA with slab thickness of $20 \mathrm{~mm}$ (white arrows and black arrowheads) (C). Transcallosal connections to cortical arteries (white arrowheads) not detected by DSA are poorly visualized on MPRAGE (D).

\section{Morphology of DSCNs}

DSCNs had pathways connecting to 2 cortical vessels: either the peripheral MCA or the anterior cerebral artery. Most anastomoses were detected between either choroidal arteries or striate arteries and cortical arteries directly or via medullary arteries (type a: Fig 2). Septal transcallosal anastomoses between choroidal arteries and pericallosal arteries were also identified (type b: Fig 2).

Collateral networks among major trunk vessels (MCA, anterior cerebral artery, choroidal arteries, and communicating arteries) were also identified as having a U-shaped running course. Especially, anastomoses between either choroidal arteries or the posterior communicating artery and thalamostriate arteries were located in the basal ganglia running adjacent to the ventricles (type c: Fig 3). Intrastriatal anastomoses among striatal arteries were prominent in the basal ganglia (type d: Fig 4). Intrathalamic anastomoses among thalamic arteries from posterior cerebral arteries were relatively rare (type e: Fig 5). Anastomoses among Moyamoya vessels focally proliferated in the basal cistern were detected in all patients (type f: Fig 4).

Eighteen (type a, $n=11$; type b, $n=7$ ) collateral networks connecting to cortical arteries were detected in DSA; 25 (type a, $n=16$; type b, $n=9$ ), in TOF-MRA; and 12 (type a, $n=8$; type b, $n=4$ ), in MPRAGE. Whereas 82 (type c, $n=21$; type d, $n=21$; type e, $n=8$; type f, $n=32$ ) collateral networks connecting major trunk vessels were detected in DSA; 81 (type c, $n=19$; type d, $n=$ 22; type e, $n=8$; type f, $n=32$ ) were detected in TOF-MRA; and 61 (type c, $n=9$; type d, $n=16$; type e, $n=6$; type f, $n=30$ ), in MPRAGE. 


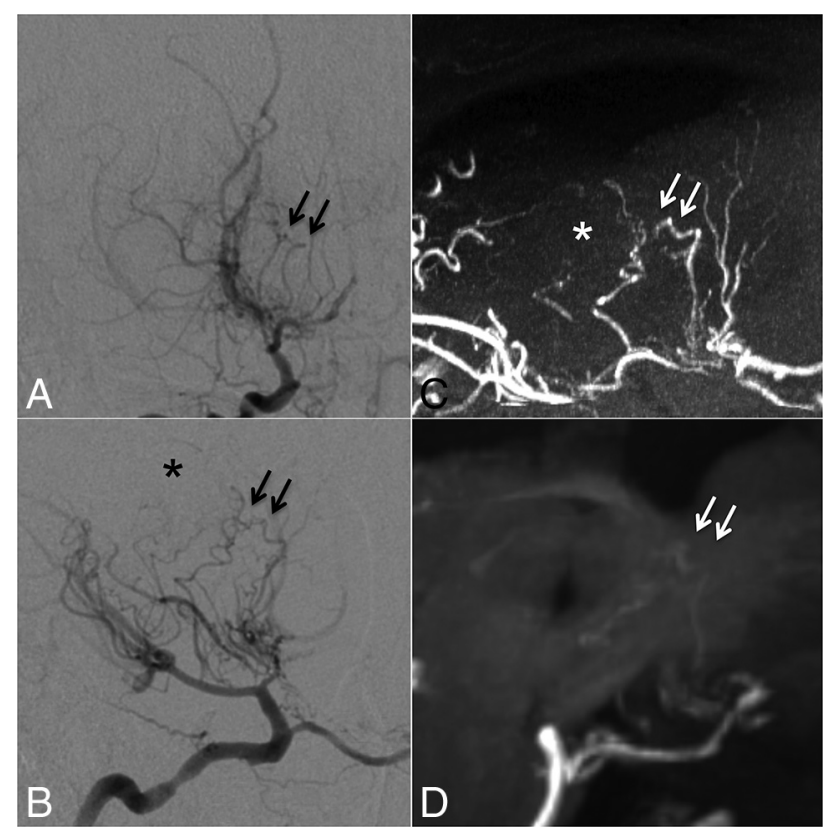

FIG 3. Case 8. Selective right internal carotid angiography (anteroposterior, $A$; right $45^{\circ}$ oblique view, $B$ ) shows anastomosis between the posterior communicating artery and the thalamostriate arteries (arrows, type c). MIP from TOF-MRA (C) demonstrates this anastomosis clearly as well as connection with the anterior choroidal artery (asterisk). The latter anastomosis could neither be detected in DSA nor visualized by MIP from MPRAGE (D).

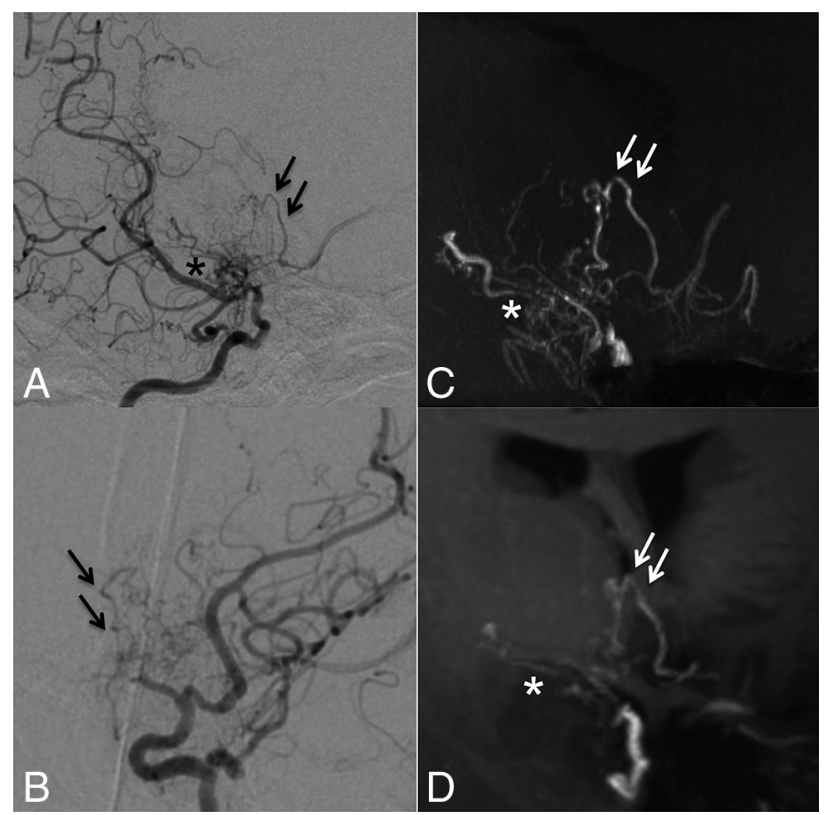

FIG 4. Case 5. Selective right internal carotid angiography (right $45^{\circ}$ oblique, $A$; left $45^{\circ}$ oblique view, $B$ ) shows intrastriatal anastomoses among thalamostriate arteries running through the periventricular space (arrows, type d). MIP from TOF-MRA (C) excellently demonstrates these networks as well as focal connections among Moyamoya vessels in the basal cistern (asterisk, type f). MIP from MPRAGE (D) moderately depicts the network; however, basal Moyamoya vessels are poorly visualized.

Regarding DSA as a clinical standard, 96 of 100 (96\%) collateral networks were delineated in TOF-MRA, and 71 of 100 (71\%), in MPRAGE. Figure 6 shows an example of an anastomosis delin-

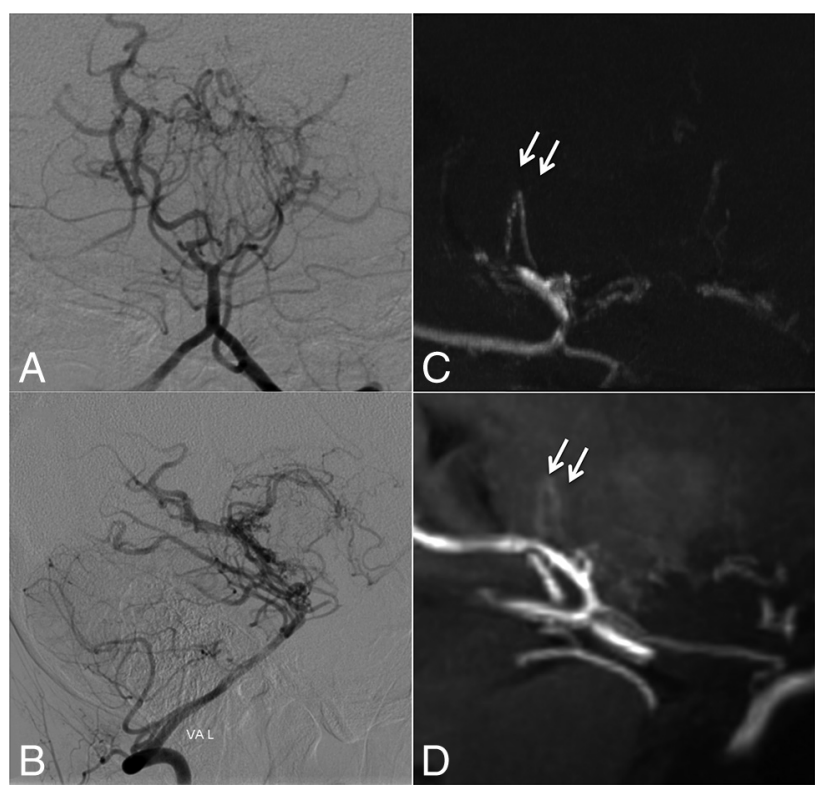

FIG 5. Case 6. Selective left vertebral angiography (anteroposterior, $A$; lateral view, $B$ ) shows no marked anastomosis. The MIP from TOFMRA (C) demonstrates excellent visualization of inner thalamic anastomosis among posterior thalamostriate arteries (white arrows, type e), whereas the visualization is only moderate on MIP from MPRAGE (D).

eated by DSA, but not by TOF-MRA or MPRAGE. Conversely, 9 anastomoses were identified by MRA (TOF: $n=9$; MPRAGE: $n=$ 3 ) that were not visible in DSA images. Seven were anastomoses with cortical arteries, and the others were an inner-striatal and a thalamic anastomosis. These DSA false-negative cases were presumably due to contrast washout and limited viewing angles during the DSA examination. Delineation of DSCNs was comparable between TOF-MRA and DSA $(P=.25)$; however, both were better than MPRAGE $(P<.001)$. The morphologic characteristics and detected number of DSCNs as well as the image quality of each imaging technique are summarized in the Table.

\section{Image-Quality Comparison among DSA, TT TOF-MRA, and MPRAGE}

Image quality of 100 collateral pathways detected on DSA (18 connecting to cortical arteries and 32 to major trunk vessels) was evaluated. In collateral pathways to cortical arteries, image quality in DSA, TOF-MRA, and MPRAGE was 4.0 (SEM = 0.28; range, $3-5), 4.8($ SEM $=0.10$; range, $3-5)$, and $2.4($ SEM $=0.35$; range, $1-5)$, respectively. The image quality of TOF-MRA proved to be significantly better than that of DSA $(P=.02)$ or MPRAGE $(P<$ $.001)$; the image quality of DSA was better than that of MPRAGE $(P=.01)$. For anastomoses to major trunk vessels, image quality of DSA and 7T TOF-MRA was comparable, $4.6(\mathrm{SEM}=0.07$; range, $2-5)$ versus 4.4 (SEM $=0.12$; range, $3-5)(P=.07)$; however, both were better than MPRAGE $2.7(\mathrm{SEM}=0.13$; range, $1-5)(P<.001)$.

\section{DISCUSSION}

This study visualized collateral networks in MMA with ultrahigh-field 7T MRA. The high spatial resolution of the applied 7T TOF-MRA sequence allowed comparable visualization of collat- 
eral networks in MMA with standard selective DSA. The spatial resolution of conventional DSA is as high as $0.1 \mathrm{~mm}^{2}$; $^{35}$ however, dynamic flow alterations and overlapping vessels can impair clear identification of small collateral networks. The lack of anatomic landmarks can further hamper understanding of the complex angioarchitecture of these networks. In contrast, MRA provides high-resolution 3D vessel delineation (TOF-MRA, $0.22 \times 0.22 \times$ $0.41 \mathrm{~mm}^{3}$ in this study) in combination with visualization of adjacent brain structures, helping to comprehend the network morphology and its distinct location. Therefore, the current clinical standard DSA is complemented by 7T MR imaging.

In the presented study, 9 collateral networks not visible on DSA could be detected by 7T MRA, with most $(n=7)$ being collateral connections to cortical vessels. The impaired visualiza-

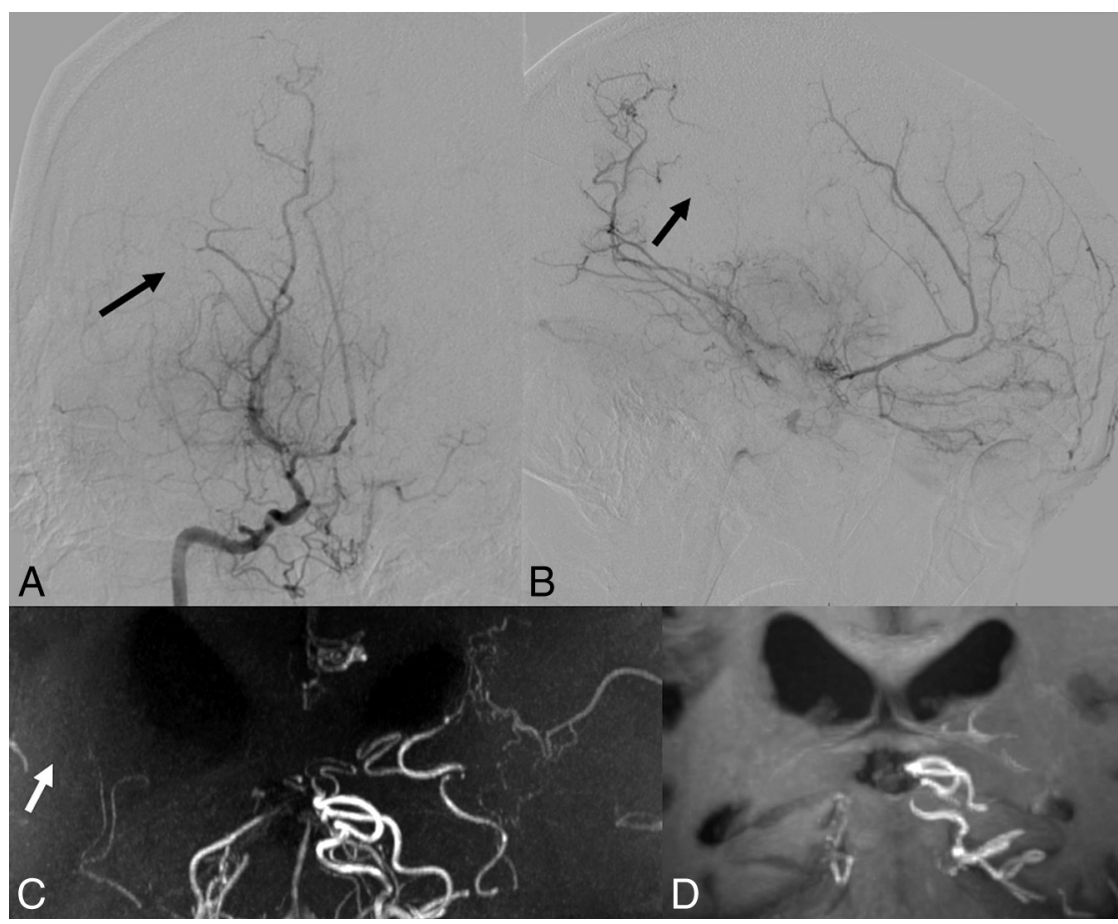

FIG 6. Case 10. Selective right vertebral angiography (anteroposterior, $A$; lateral view, $B$ ) shows a tiny, barely visible anastomosis (black arrows) between the posterior thalamostriate artery and a medullary-cortical artery. MIP from TOF-MRA (C) and MIP from MPRAGE (D) do not delineate the anastomosis. The white arrow $(C)$ indicates the supposed anatomic location of the anastomosis. tion in DSA might be explained by flow interactions of various anastomotic collateral vessels (ie, leptomeningeal or durocortical collateral networks) predominantly in peripheral territories. A second explanation is visualization impairment due to overlapping vessel structures hiding small collateral networks. As reported in previous studies, TOF-MRA was significantly better for visualization of submillimeter vessels than nonenhanced MPRAGE. ${ }^{24-27}$ The main disadvantage of the MPRAGE sequence was the lower spatial resolution, which restricted detection of small collateral networks. Furthermore, visualization of the complete collateral network was also impaired in most cases. Although the applied MPRAGE sequence successfully visualized some collateral networks, it was inferior to 7T TOF-MRA and DSA and does not seem suitable for evaluation of the complex angioarchitecture in MMA.

In addition to the combination of high-resolution vessel delineation and depiction of adjacent brain structures by 7T MRA, application of MIP processing allows visualization of collateral networks comparable with superselective DSA. Nevertheless, collateral vessels in MMA have a very complex angioarchitecture and are difficult to investigate, even with superselective DSA. Recently, Baltsavias et $\mathrm{al}^{32-34}$ first and precisely demonstrated the collateral circulations in pediatric Moyamoya disease by superselective DSA with microcatheters. However, superselective DSA is accompanied by considerable risks for cerebral hemorrhage because of vessels that might be injured by the microcatheter, especially in fragile Moyamoya collaterals. Besides being noninvasive, 1 further major advantage of 7T MRA MIP over superselective angiography is the possibility of unrestricted $3 \mathrm{D}$ rotations.

The excellent depiction of the angioarchitecture by 7T TOF-MRA in combination with MIP allowed the establish-

Comparison of 7T MRA and DSA regarding the number of detected collateral networks and image quality for anastomoses to major trunk vessels and collateral pathways to cortical vessels

\begin{tabular}{|c|c|c|c|c|}
\hline Type & Connecting Vessels in Collateral Networks & DSA & TOF-MRA & MPRAGE \\
\hline \multicolumn{5}{|c|}{$\begin{array}{l}\text { Collateral networks connecting } \\
\text { to cortical arteries }\end{array}$} \\
\hline a & Striate/choroidal arteries and medullary-cortical arteries & 11 & 16 & 8 \\
\hline \multirow[t]{3}{*}{$b$} & Striate/choroidal arteries and the pericallosal arteries & 7 & 9 & 4 \\
\hline & Total & 18 & 25 & 12 \\
\hline & Image quality $(n=18)^{a}$ (mean) (SEM) (range) & $4.0(0.26,3-5)$ & $4.8(0.10,3-5)$ & $2.4(0.35,1-5)$ \\
\hline \multicolumn{5}{|c|}{$\begin{array}{l}\text { Collateral networks connecting } \\
\text { to major trunk vessels }\end{array}$} \\
\hline c & Choroidal arteries or PcomA and thalamostriate arteries & 21 & 19 & 9 \\
\hline$d$ & Intrastriatal arteries & 21 & 22 & 16 \\
\hline e & Intrathalamic arteries & 8 & 8 & 6 \\
\hline \multirow[t]{3}{*}{$f$} & Moyamoya vessels in the basal cistern & 32 & 32 & 30 \\
\hline & Total & 82 & 81 & 61 \\
\hline & Image quality $(n=82)^{a}$ (mean) (SEM) (range) & $4.6(0.07,2-5)$ & $4.4(0.12,3-5)$ & $2.7(0.13,1-5)$ \\
\hline
\end{tabular}

Note:-PcomA indicates posterior communicating artery.

${ }^{a}$ Image quality was evaluated in collateral networks detected by DSA as a clinical standard using a 5-point scale. 
ment of a comprehensive classification of collateral networks in MMA. The classification comprises 2 main subtypes: collateral networks connecting the circle of Willis with cortical arteries (remote type) and collateral networks between major trunk vessels (local type). The latter are characterized by their U-shaped running course. In total, 6 distinct collateral network types were identified. So far, the clinical relevance of collateral vessels has been indirectly evaluated by depiction of dilated branching arteries (ie, of the posterior communicating artery and/or the anterior choroidal artery). ${ }^{6-8}$ Recently, 3T flow-sensitive black-blood MRA allowed Funaki et al ${ }^{18}$ to specify one of the deeply seated collateral pathways adjacent to the ventricles, the so-called "periventricular anastomosis" between either thalmo-perforating or choroidal arteries and medullary arteries. They suggested that this pathway is associated with vessel fragility and hemorrhagic presentation at onset in Moyamoya disease. ${ }^{9}$ A recent 7T TOF-MRA study on intraventricular submillimeter microaneurysms in collateral networks branching from the posterior choroidal artery supports this hypothesis. ${ }^{36}$ Although the clinical relevance of the different collateral networks remains unclear, the presented classification can improve our understanding of the complex pathophysiology of MMA and might serve as a follow-up marker, especially after bypass surgery.

With regard to MRA, the better delineation of Moyamoya vessels in stronger magnets has been previously reported. ${ }^{16,17,37}$ However, these studies only analyzed summation of high-signalintensity areas associated with Moyamoya vessels or pathophysiologic major trunks at the terminal portion of the internal carotid artery. ${ }^{16,17,37}$ Recent studies using ultra-high-field 7T MRA have enhanced the diagnostic accuracy of this neurovascular imaging technique to delineate microvascular structures and even vessel walls. $^{20-23}$ The presented study used dedicated MRA sequences to identify possible collateral networks with up to 2.4- to 3.2-fold higher resolution than in recently reported studies on MMA using 7T MRA. ${ }^{17,37}$ This study shows the possible clinical application of 7T MRA to identify submillimeter collateral networks in MMA. Therefore, further studies including larger patient numbers at different Suzuki stages are warranted to investigate the clinical importance of each collateral pathway.

Nevertheless, the presented study has some limitations. The main limitation is the relatively small number of patients $(n=15)$ with MMA and different Suzuki stages. Ratings might be biased due to memory of collateral pathways from a previous evaluation. However, due to long intervals between reading sessions and the complex vascular anatomy, memory bias seemed to be a minor issue. Enlisting a substantially larger number of patients with MMA for a 7T MR imaging study remains challenging due to the low incidence of MMA and the limited availability of 7T MR imaging systems. A direct comparison of 7T MRA with superselective DSA would have been beneficial but was rejected because of the increased risk for the patients. Furthermore, conclusions derived from comparing high-resolution TOF-MRA with much lower resolution MPRAGE are limited, but scanning time for a MPRAGE sequence with comparable resolution would, by far, have exceeded typical patient tolerance. A lower resolution MPRAGE sequence was therefore included in the study protocol as previously applied in several cerebrovascular studies including
MMA studies. ${ }^{17,38}$ Finally, the study design did not include MRA at conventional field strengths ( $1.5 \mathrm{~T}$ or $3 \mathrm{~T}$ ) due to heterogeneous previous imaging sessions, but numerous recent studies have already shown the superiority of $7 \mathrm{~T}$ over $3 \mathrm{~T}$ TOF-MRA for delineation of submillimeter vessels. ${ }^{17,37}$

\section{CONCLUSIONS}

In the presented study, 7T MRA could delineate DSCNs in MMA in a clinical setting. With respect to submillimeter collateral vessels, visualization by 7T TOF-MRA was comparable with that in DSA. For further morphologic and pathophysiologic research on submillimeter collateral networks in MMA, 7T TOF-MRA is a very promising imaging technique.

Disclosures: Markus Kraemer-UNRELATED: Grants/Grants Pending: Novartis, German Neurological Society*; Payment for Lectures Including Service on Speakers Bureaus: Merck Serono; Travel/Accommodations/Meeting Expenses Unrelated to Activities Listed: Merck Serono, Novartis, Shire, Genzyme. Karsten H. WredeRELATED: Grant: Programm zur internen Forschungsförderung Essen. *Money paid to the institution.

\section{REFERENCES}

1. Suzuki J, Takaku A. Cerebrovascular “moyamoya” disease: disease showing abnormal net-like vessels in base of brain. Arch Neurol 1969;20:288-99 CrossRef Medline

2. Research Committee on the Pathology and Treatment of Spontaneous Occlusion of the Circle of Willis; Health Labour Sciences Research Grant for Research on Measures for Infractable Diseases. Guidelines for diagnosis and treatment of moyamoya disease (spontaneous occlusion of the circle of Willis). Neurol Med Chir (Tokyo) 2012;52:245-66 CrossRef Medline

3. Bersano A, Guey S, Bedini G, et al; European Moyamoya Disease Initiative. Research progresses in understanding the pathophysiology of Moyamoya disease. Cerebrovasc Dis 2016;41:105-18 CrossRef Medline

4. Bedini G, Blecharz KG, Nava S, et al. Vasculogenic and angiogenic pathways in Moyamoya disease. Curr Med Chem 2016;23:315-45 CrossRef Medline

5. Yamada I, Matsushima Y, Suzuki S. Moyamoya disease: diagnosis with three-dimensional time-of-flight MR angiography. Radiology 1992;184:773-78 CrossRef Medline

6. Morioka M, Hamada J, Kawano T, et al. Angiographic dilatation and branch extension of the anterior choroidal and posterior communicating arteries are predictors of hemorrhage in adult moyamoya patients. Stroke 2003;34:90-95 CrossRef Medline

7. Strother MK, Anderson MD, Singer RJ, et al. Cerebrovascular collaterals correlate with disease severity in adult North American patients with Moyamoya disease. AJNR Am J Neuroradiol 2014;35: 1318-24 CrossRef Medline

8. Jang DK, Lee KS, Rha HK, et al. Clinical and angiographic features and stroke types in adult moyamoya disease. AJNR Am JNeuroradiol 2014;35:1124-31 CrossRef Medline

9. Funaki T, Takahashi JC, Yoshida K, et al. Periventricular anastomosis in moyamoya disease: detecting fragile collateral vessels with MR angiography. J Neurosurg 2016;124:1766-72 CrossRef Medline

10. Kronenburg A, Braun KP, van der Zwan A, et al. Recent advances in moyamoya disease: pathophysiology and treatment. Curr Neurol Neurosci Rep 2014;14:423 CrossRef Medline

11. Kraemer M, Diehl RR, Diesner F, et al. Differential diagnosis between cerebral ischemia, focal seizures and limb shaking TIAs in moyamoya disease. Br J Neurosurg 2012;26:896-98 CrossRef Medline

12. Kraemer M, Heienbrok W, Berlit P. Moyamoya disease in Europeans. Stroke 2008;39:3193-200 CrossRef Medline

13. Krischek B, Kasuya H, Khan N, et al. Genetic and clinical characteristics of Moyamoya disease in Europeans. Acta Neurochir Suppl 2011;112:31-34 CrossRef Medline 
14. Ganesan V. Moyamoya: to cut or not to cut is not the only question-a paediatric neurologist's perspective. Dev Med Child Neurol 2010;52:10-13 CrossRef Medline

15. Kraemer M, Lee SI, Ayzenberg I, et al. Headache in Caucasian patients with moyamoya angiopathy: a systematic cohort study. Cephalalgia 2017;160:137-41 CrossRef Medline

16. Fushimi Y, Miki Y, Kikuta K, et al. Comparison of 3.0- and 1.5-T three-dimensional time-of-flight MR angiography in moyamoya disease: preliminary experience. Radiology 2006;239:232-37 CrossRef Medline

17. Deng X, Zhang Z, Zhang Y, et al. Comparison of 7.0- and 3.0-T MRI and MRA in ischemic-type moyamoya disease: preliminary experience. J Neurosurg 2016;124:1716-25 CrossRef Medline

18. Funaki T, Fushimi Y, Takahashi JC, et al. Visualization of periventricular collaterals in Moyamoya disease with flow-sensitive blackblood magnetic resonance angiography: preliminary experience. Neurol Med Chir (Tokyo) 2015;55:204-09 CrossRef Medline

19. Cho ZH, Kang CK, Han JY, et al. Observation of the lenticulostriate arteries in the human brain in vivo using 7.0T MR angiography. Stroke 2008;39:1604-06 CrossRef Medline

20. van der Kolk AG, Zwanenburg JJ, Brundel M, et al. Intracranial vessel wall imaging at 7.0-T MRI. Stroke 2011;42:2478-84 CrossRef Medline

21. Kleinloog R, Korkmaz E, Zwanenburg JJ, et al. Visualization of the aneurysm wall: a 7.0-Tesla magnetic resonance imaging study. $\mathrm{Neu}$ rosurgery 2014;75:614-22; discussion 622 CrossRef Medline

22. Matsushige T, Chen B, Ringelstein A, et al. Giant intracranial aneurysms at 7T MRI. AJNR Am J Neuroradiol 2016;37:636-41 CrossRef Medline

23. Matsushige T, Chen B, Dammann P, et al. Microanatomy of the subcallosal artery: an in-vivo $7 \mathrm{~T}$ magnetic resonance angiography study. Eur Radiol 2016;26:2908-14 CrossRef Medline

24. Bouvy WH, Biessels GJ, Kuijf HJ, et al. Visualization of perivascular spaces and perforating arteries with $7 \mathrm{~T}$ magnetic resonance imaging. Invest Radiol 2014;49:307-13 CrossRef Medline

25. Conijn MM, Hendrikse J, Zwanenburg JJ, et al. Perforating arteries originating from the posterior communicating artery: a 7.0-Tesla MRI study. Eur Radiol 2009;19:2986-92 CrossRef Medline

26. Harteveld AA, De Cocker LJ, Dieleman N, et al. High-resolution postcontrast time-of-flight MR angiography of intracranial perforators at 7.0 Tesla. PLoS One 2015;10:e0121051 CrossRef Medline
27. Wrede KH, Johst S, Dammann P, et al. Improved cerebral time-offlight magnetic resonance angiography at 7 Tesla: feasibility study and preliminary results using optimized venous saturation pulses. PLoS One 2014;9:e106697 CrossRef Medline

28. Johst S, Wrede KH, Ladd ME, et al. Time-of-flight magnetic resonance angiography at $7 \mathrm{~T}$ using venous saturation pulses with reduced flip angles. Invest Radiol 2012;47:445-50 CrossRef Medline

29. Wrede KH, Johst S, Dammann P, et al. Caudal image contrast inversion in MPRAGE at 7 Tesla: problem and solution. Acad Radiol 2012;19:172-78 CrossRef Medline

30. Umutlu L, Theysohn N, Maderwald S, et al. 7 Tesla MPRAGE imaging of the intracranial arterial vasculature: nonenhanced versus contrast-enhanced. Acad Radiol 2013;20:628-34 CrossRef Medline

31. Wrede KH, Dammann P, Johst S, et al. Non-enhanced MR imaging of cerebral arteriovenous malformations at 7 Tesla. Eur Radiol 2016; 26:829-39 CrossRef Medline

32. Baltsavias G, Khan N, Valavanis A. The collateral circulation in pediatric moyamoya disease. Childs Nerv Syst 2015;31:389-98 CrossRef Medline

33. Baltsavias G, Khan N, Filipce V, et al. Selective and superselective angiography of pediatric moyamoya disease angioarchitecture in the posterior circulation. Interv Neuroradiol 2014;20:403-12 CrossRef Medline

34. Baltsavias G, Valavanis A, Filipce V, et al. Selective and superselective angiography of pediatric moyamoya disease angioarchitecture: the anterior circulation. Interv Neuroradiol 2014;20:391-402 CrossRef Medline

35. Chang W, Huang M, Chien A. Emerging techniques for evaluation of the hemodynamics of intracranial vascular pathology. Neuroradiol J 2015;28:19-27 Medline

36. Matsushige T, Kraemer M, Schlamann M, et al. Ventricular microaneurysms in Moyamoya angiopathy visualized with 7T MR angiography. AJNR Am J Neuroradiol 2016;37:1669-72 CrossRef Medline

37. Dengler NF, Madai VI, Wuerfel J, et al. Moyamoya vessel pathology imaged by ultra-high-field magnetic resonance imaging at 7.0 T.J Stroke Cerebrovasc Dis 2016;25:1544-51 CrossRef Medline

38. Oh BH, Moon HC, Baek HM, et al. Comparison of 7T and 3T MRI in patients with moyamoya disease. Magn Reson Imaging 2017;37: 134-38 CrossRef Medline 